\title{
Practical aspects of stochastic dynamic tidal modelling
}

\author{
A. W. Heemink \\ Data Processing Division, Rijkswaterstaat, Nijverheidsstraat 1, \\ 2280 HV Rijswik (Z. H.), The Netherlands
}

\begin{abstract}
Kalman filtering for stochastic dynamic tidal models, is a hyperbolic filtering problem. The questions of observability and stability of the filter as well as the effects of the finite difference approximation on the filter performance are studied. The degradation of the performance of the filter, in case an erroneous filter model is used, is investigated. In this paper we discuss these various practical aspects of the application of Kalman filtering for tidal flow identification problems. Filters are derived on the basis of the linear shallow water equations. Analytical methods are used to study the performance of the filters under a variety of circumstances.
\end{abstract}

Key words: shallow water equations, Kalman filtering, data assimilation, observability.

\section{Introduction}

Kalman filtering has turned out to be a powerful new tool for tidal prediction (e.g. Budgell and Unny 1980; Brummelhuis, De Jong and Heemink 1984; Heemink 1986). Kalman filtering is a data assimilation technique that incorporates measurements into a numerical model (Parrish and Cohn 1985, Miller 1986, Budgell 1986, Heemink 1988). In most common data assimilation approaches some estimates of the error statistics of the numerical model are used to correct the results of the model using on-line measurements. Employing Kalman filtering these error statistics are determined by using the stochastic extension of the numerical model. In this way the correction produced by the Kalman filter is guaranteed to be consistent with this stochastic model, even in the case of very irregular flow patterns caused by complicated geometries.

In rivers and estuaries a one-dimensional representation of the water movement is adequate. Based on the work of Heemink and De Jong (1982) and of Brummelhuis, De Jong and Heemink $(1984,1988)$, a Kalman filter procedure has been developed for one-dimensional tidal filtering. It is based on the non-linear St. Venant equations describing the long wave motion in an open channel. By means of the Preissmann four-point scheme these equations are discretised and by introducing a system noise process into the resulting difference equations, a discrete system representation of the stochastic-dynamic model is obtained. By employing an extended Kalman filter procedure, the state of the system as well as some uncertain parameters in the model, such as the bottom friction coefficient and the wind stress coefficient, are identified using measurements of the water-level only. The 
resulting filter is applicable to a wide range of practical shallow water flow problems. A number of stochastic-dynamic tidal models have been implemented in the Netherlands, e.g. of the Eastern Scheldt estuary, the Western Scheldt estuary and the Rhine-Scheldt channel.

In employing a Kalman filter procedure for tidal prediction, it is important to study questions of observability and stability of the filter as well as the effects of the finite difference approximation on the filter performance. As known (Jazwinski 1970 ), one of the major problem in the application of Kalman filtering is that the noise statistics have to be specified. This knowledge is very poor in case of environmental systems and, consequently, it is important to get insight into the degradation of the performance of the filter in case erroneous noise statistics are used. In this paper we discuss these practical aspects of Kalman filtering for hyperbolic-type identification problems. We derive filters on the basis of the linear one-dimensional equations. In this case it is possible to employ analytical methods to investigate the performance of the filters. In Section 2 we recall some basic aspects of the shallow water equations and discuss the questions of observability and filter divergence from a continuum physics viewpoint. Section 3 is devoted to the numerical approximation of the shallow water equations, while in Section 4 the choice of the noise statistics is discussed. A specific filtering problem is solved in Section 5. For this case the sensitivity of the filter performance is studied with respect to the choice of the finite difference scheme as well as modelling errors.

\section{The one-dimensional shallow water equations}

The linear one-dimensional shallow water equations used throughout this paper are (Abbott 1979):

$$
\frac{\partial \mathbf{F}}{\partial t}+C_{1} \frac{\partial \mathbf{F}}{\partial x}+C_{2} \mathbf{F}=0
$$

where:

$$
\mathbf{F}=\left[\begin{array}{ll}
u & h
\end{array}\right]^{T}, \quad C_{1}=\left[\begin{array}{ll}
0 & g \\
D & 0
\end{array}\right], \quad C_{2}=\left[\begin{array}{ll}
\lambda & 0 \\
0 & 0
\end{array}\right]
$$

and $h=$ water-level, $u=$ water velocity, $D=$ depth of the water, $g=$ acceleration of gravity, $\lambda=\mu / D$ where $\mu=$ linear bottom friction coefficient. The water movement is completely described by these equations, provided that initial and boundary conditions are given.

Resolving the question of the observability of the hyperbolic system (1) is important in choosing measurement locations in the spatial domain and to get insight into the predictive capabilities of the Kalman filter. We discuss this question using the definition of observability of a distributed parameter system proposed by Goodson and Klein (1970) who have investigated the observability from a continuum physics viewpoint. Their definition is based on the theory of the existence and uniqueness of the solution to a partial-differential equation.

Consider Eq. (1) and assume measurements are available over the time interval $\left[t_{0}, t_{p}\right]$ at some locations. This system is said to be $\mathrm{G}-\mathrm{K}$-observable in the domain $\Omega_{o}\left(x, t, t_{p}\right)$ if and only if a unique solution $u(x, t), h(x, t)$ in $\Omega_{o}\left(x, t, t_{p}\right)$ is established by the measurements taken and by the boundary conditions. G-K-observability may also be established by the measurements alone without knowledge of the boundary conditions. It can be shown that for linear ordinary differential equations G-K-observability reduces to observability as defined by Kalman (1960) (Goodson and Klein 1970). 


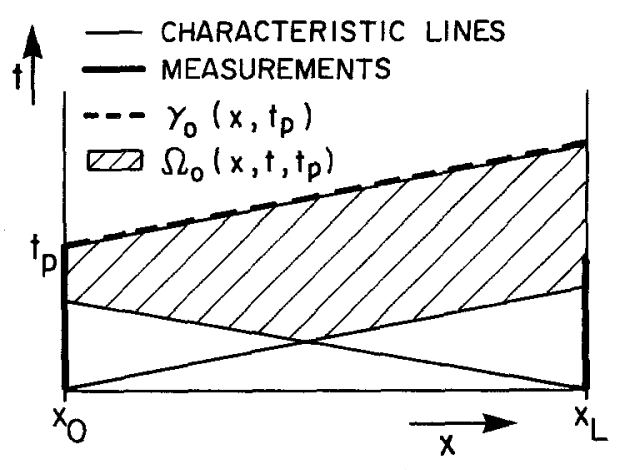

Figure 1

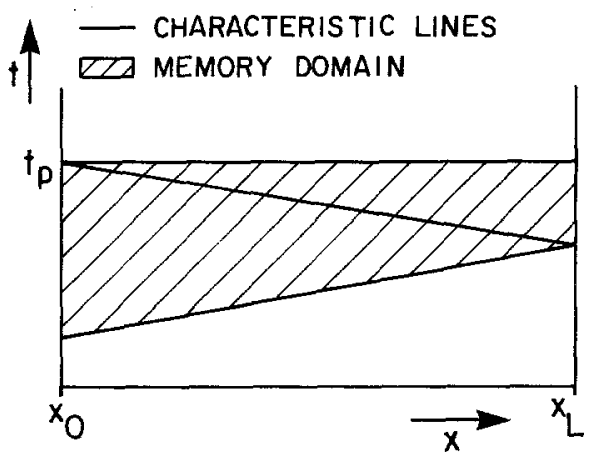

Figure 2

Figure 1. Construction of the observability domain and the prediction horizon

Figure 2. Construction of the memory domain

The concept of the G-K-observability domain $\Omega_{o}\left(x, t, t_{p}\right)$ is used to determine a prediction horizon at time $t_{p}$. This prediction horizon $\gamma_{o}\left(x, t_{p}\right)$ of the filter establishes in each point of the spatial domain the period over which predictions are uniquely determined by the measurements taken up to time $t_{p}$ and, if known, by the boundary conditions. One expects that for periods greater than $\gamma_{o}\left(x, t_{p}\right)$ the prediction is inaccurate. In Fig. 1 an example of the G-K-observability domain $\Omega_{o}\left(x, t, t_{p}\right)$ and the prediction horizon $\gamma_{o}\left(x, t_{p}\right)$ are given. Here we consider the model (1) without friction and prescribe the water-level at $x=x_{0}$ and at $x=x_{L}$ the condition $u=0$.

Now we consider again the system (1) without bottom friction and with boundary conditions as given and we construct the set of characteristic lines that intersect the line $t=t_{p}, x \in\left[x_{0}, x_{L}\right]$. This domain is considered as the memory of the filter (see Fig. 2). Since the water-levels and velocities outside the memory domain does not affect $u\left(x, t_{p}\right)$ and $h\left(x, t_{p}\right), x \in\left[x_{0}, x_{L}\right]$, only measurements taken inside this domain are useful to estimate $u\left(x, t_{p}\right)$ and $h\left(x, t_{p}\right), x \in\left[x_{0}, x_{L}\right]$. The most recent observations are used to determine these estimates.

With respect to the problem of filter divergence the construction of the memory domain is important. The problem of divergence is caused by the fact that the stochastic model used by the filter is never perfect. When the filter operates with a great amount of data, it may learn the state "too well". The error covariance, as well as, the filter gain become very small and subsequent observations have little effect on the estimate. In essence, filter divergence is due to the fact that old observations are weighted too heavily. They can be valueless when predicted over long periods of time through an erroneous model. Numerous ad hoc remedies to the problem of filter divergence have been developed (Jazwinski 1970). A wellknown approach which has a sound theoretical basis is the limited memory filter. This filter produces estimates of the state only on the basis of the $N$ most recent observations. $N$ should be chosen such that the model is an adequate approximation to the real system over the time interval $\left[t_{k-N}, t_{k}\right]$. By constructing the memory domain of the system (1) without bottom friction (see Fig. 2), one can show that the memory of this filter is limited in nature. Therefore, filter 


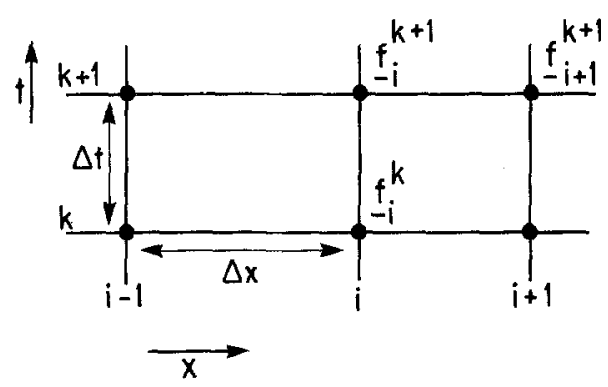

Figure 3. The grid

divergence is not likely to occur. This important conclusion holds for many practical filtering problems of the hyperbolic type.

\section{Discretisation}

To develop a discrete system representation Eq. (1) is discretised. Defining a grid (see Fig. 3), we consider two different finite differences schemes. Firstly, we employ the implicit four-point Preissmann scheme (Liggett and Cunge 1975):

$\frac{1}{\Delta t}\left(\mathbf{F}_{i+1 / 2}^{k+1}-\mathbf{F}_{i+1 / 2}^{k}\right)+\frac{1}{\Delta x} C_{1}\left(\mathbf{F}_{i+1}^{k+\theta}-\mathbf{F}_{i}^{k+\theta}\right)+C_{2} \mathbf{F}_{i+1 / 2}^{k+1 / 2}=0$,

where $\mathbf{F}_{i}^{k}$ is the approximation of $\mathbf{F}(i \Delta x, k \Delta t)$ and

$\mathbf{F}_{i+1 / 2}^{k}=1 / 2\left(\mathbf{F}_{i+1}^{k}+\mathbf{F}_{i}^{k}\right) ; \quad \mathbf{F}_{i}^{k+\theta}=\theta \mathbf{F}_{i}^{k+1}+(1-\theta) \mathbf{F}_{i}^{k}$.

Here $\theta$ is a weighting factor. For the special case of $\lambda=0$ it is easy to show that the finite difference scheme (2) is a consistent second-order approximation of the partial-differential equation:

$\frac{\partial \mathbf{F}}{\partial t}+C_{1} \frac{\partial \mathbf{F}}{\partial x}-(\theta-0.5) \Delta t C_{1}^{2} \frac{\partial^{2} \mathbf{F}}{\partial x^{2}}=0$.

Comparing Eqs. (1) and (3) we see that the original Eq. (1) is solved with secondorder accuracy only for $\theta=0.5$. By choosing $0.5<\theta \leq 1.0$ one introduces numerical diffusion.

The second finite difference scheme considered is the explicit Lax-Wendroff scheme (Richtmyer and Morton 1967):

$$
\begin{aligned}
\mathbf{F}_{i}^{k+1}=\mathbf{F}_{i}^{k} & -\frac{\Delta t}{2 \Delta x} C_{1}\left(\mathbf{F}_{i+1}^{k}-\mathbf{F}_{i-1}^{k}\right) \\
& +\left(\frac{\Delta t}{\Delta x} C_{1}\right)^{2}\left(\mathbf{F}_{i+1}^{k}-2 \mathbf{F}_{i}^{k}+\mathbf{F}_{i-1}^{k}\right)-\Delta t C_{2}\left(\mathbf{F}_{i-1}^{k}+\mathbf{F}_{i+1}^{k}\right) .
\end{aligned}
$$

This scheme is of second-order accuracy.

To analyze the stability and to gain insight into the dissipative properties of the schemes for initial value problems, a Von Neumann stability analysis is employed. Consider the behaviour of the Fourier integral: 
$\mathbf{F}_{i}^{k}=\int_{-0.5 \Delta x}^{0.5 \Delta x} \zeta^{k}(\ell) e^{j 2 \pi \ell i \Delta x} d \ell$

under the operation given by Eq. (2). Here $j$ is the imaginary unit, $\ell$ is the wave number and $0.5 \Delta x$ is the highest wave number that can be resolved. Substituting this Fourier integral into the Preissmann scheme (2) yields:

$A(\ell) \zeta^{k+1}(\ell)=B(\ell) \zeta^{k}(\ell)$,

where:

$A(\ell)=I+\frac{1}{2} \Delta t C_{2}+2 j \frac{\Delta t}{\Delta x} \theta \tan (\pi \ell \Delta x) C_{1} \quad$ and

$B(\ell)=I-\frac{1}{2} \Delta t C_{2}-2 j \frac{\Delta t}{\Delta x}(1-\theta) \tan (p l d x) C_{1}$.

Equation (6) can be rewritten as:

$\zeta^{k+1}(\ell)=G(\ell) \zeta^{k}(\ell)$

where $G(\ell)=A(\ell)^{-1} B(\ell)$ is the amplification matrix of the finite difference scheme. This matrix amplifies the value of $\zeta^{k}(\ell)$ over a time step $\Delta t$. In case $0.5<\theta \leq 1$ we have for the eigenvalues $g_{1,2}(\ell)$ of $G(\ell)$ that $\left|g_{1,2}(\ell)\right|<1$ and consequently the scheme is unconditionally stable.

Analogously the amplification matrix of the Lax-Wendroff scheme is given by:

$$
\begin{aligned}
G(\ell)=I & -j \frac{\Delta t}{\Delta x} C_{1} \sin (2 \pi \ell \Delta x) \\
& -\left(\frac{\Delta t}{\Delta x} C_{1}\right)^{2}(1-\cos (2 \pi \ell \Delta x))-\Delta t C_{2} \cos (2 \pi \ell \Delta x),
\end{aligned}
$$

yielding the sufficient condition for stability:

$\sqrt{(1 / 2) \lambda \Delta t}<C r<\sqrt{1+(1 / 2) \lambda \Delta t}$,

with $C r=\sqrt{g D} \cdot \Delta t / \Delta x$ the Courant number.

\section{On the choice of the system noise}

The tidal movement is not perfectly described by the finite difference equations. Therefore, we embed these equations into a stochastic environment by adding system noise. For the Preissmann scheme we obtain:

$\frac{1}{\Delta t}\left(\mathbf{F}_{i+1 / 2}^{k}-\mathbf{F}_{i+1 / 2}^{k+1}\right)+\frac{1}{\Delta x} C_{1}\left(\mathbf{F}_{i+1}^{k+\theta}-\mathbf{F}_{i}^{k+\theta}\right)+C_{2} \mathbf{F}_{i+1 / 2}^{k+1 / 2}+\mathbf{W}_{i}^{k}=0$,

where $\mathbf{W}_{i}^{k}=\left[W m_{i}^{k} W c_{i}^{k}\right]^{T}$ is the system noise. The noise processes $W m_{i}^{k}$ and $W c_{i}^{k}$ are associated with the uncertainty of the momentum equation and the continuity equation respectively. The covariance of $\mathbf{W}_{i}^{k}$ is chosen as:

$E\left\{\begin{array}{ll}\mathbf{W}_{i 1}^{k 1} & \mathbf{W}_{i 2}^{k 2}\end{array}\right\}= \begin{cases}Q_{i 1, i 2}\left(k_{1}\right), & k_{1}=k_{2} \\ 0, & k_{1} \neq k_{2}\end{cases}$

By introducing the system noise, we associate this random process with the discretised momentum and continuity equations. However, when employing an implicit scheme it is also possible to introduce the system noise process after the finite difference equations have been solved and $\mathbf{F}_{i}^{k}$ is known explicitly. In this 
case the system noise process is associated with the uncertainty of the computed water-levels and velocities.

For the Lax-Wendroff scheme we introduce the system noise as follows:

$$
\begin{aligned}
\mathbf{F}_{i}^{k+1}=\mathbf{F}_{i}^{k} & -\frac{\Delta t}{2 \Delta x} C_{1}\left(\mathbf{F}_{i+1}^{k}-\mathbf{F}_{i-1}^{k}\right) \\
& +\left(\frac{\Delta t}{\Delta x} C_{1}\right)^{2}\left(\mathbf{F}_{i+1}^{k}-2 \mathbf{F}_{i}^{k}+\mathbf{F}_{i-1}^{k}\right)-\Delta t C_{2}\left(\mathbf{F}_{i-1}^{k}+\mathbf{F}_{i+1}^{k}\right)+\Delta t \mathbf{W}_{i}^{k}
\end{aligned}
$$

The system noise represents the errors of the corresponding deterministic model. It includes the variability in the natural system, e.g., due to turbulent effects and model structure errors such as neglected non-linearities or wrong parameter values, as well as, errors caused by the discretisation of the partial-differential equations. Unfortunately, in practice very little is known about the statistics of the system noise. In some cases (Jazwinski 1970) it is possible to use a systematic approach to determine the covariance $Q_{i 1, i 2}(k)$. However, usually it has to be established by means of "trial and error", i.e., the filter is employed for various values of $Q_{i 1, i 2}(k)$, until one gets satisfactory filter performance.

In choosing a suitable value for $Q_{i 1, i 2}(k)$, it has to be taken into account that finite difference schemes are not able to accurately represent short waves, i.e., waves with a wavelength of the order of $2 \Delta x$. Therefore, in order to obtain meaningful solutions and to avoid instabilities when solving the time propagation of the estimation error covariance, the energy of these short noise waves should be limited.

Assuming the system noise is location invariant it is then described by the spatial covariance functions:

$$
\begin{aligned}
& E\left\{W m_{i 1}^{k} W m_{i 2}^{k}\right\}=\operatorname{Qm}\left(\left|i_{1}-i_{2}\right|, k\right) \\
& E\left\{W c_{i 1}^{k} \quad W c_{i 2}^{k}\right\}=\operatorname{Qc}\left(\left|i_{1}-i_{2}\right|, k\right),
\end{aligned}
$$

where $W m_{i}^{k}$ and $W c_{i}^{k}$ are supposed to be mutually independent. Neglecting boundary effects the distribution of the system noise as function of the wave number $\ell$ is given by:

$$
\begin{aligned}
& S m(\ell, k)=\Delta x \sum_{i=-\infty}^{\infty} Q m(|i|, k) e^{-j 2 \pi \ell i \Delta x} \\
& S c(\ell, k)=\Delta x \sum_{i=-\infty}^{\infty} Q c(|i|, k) e^{-j 2 \pi \ell i \Delta x}
\end{aligned}
$$

As noted before, the energy of the short waves has to be limited. However, this energy is not allowed to be too small in order to avoid that the filtering problem is ill-conditioned and that numerical difficulties due to the numerical computation using finite wordlength are likely to occur.

Finally, we note that in practice the time-varying behaviour of the statistics of noise processes is poorly known. Therefore, these processes are usually assumed to be stationary.

\section{Kalman filtering in the wave number domain}

As described in Section 3, a finite difference scheme can be transformed into the wave number domain by deriving the amplification matrix $G(\ell)$ of the scheme. Although this procedure can only be employed for linear equations and does not 
include the influence of the boundary conditions, it increases the insight into the properties of a finite difference scheme. In some special cases, this approach can be generalized to study the properties of the Kalman filter.

Suppose the propagation in time of the process $\mathbf{F}_{i}^{k}$ is described by Eq. (10) or (12), where the noise processes $W m_{i}^{k}$ and $W c_{i}^{k}$ are supposed to be location invariant, stationary and mutually independent. Observations of the water-level are available at all grid points:

$Z_{i}^{k}=M \mathbf{F}_{i}^{k}+V_{i}^{k}$,

where $M=\left[\begin{array}{ll}0 & 1\end{array}\right]$, and $V_{i}^{k}$ is the measurement noise with covariance:

$E\left\{\begin{array}{ll}V_{i 1}^{k 1} & V_{i 2}^{k 2}\end{array}\right\}= \begin{cases}r^{2}, & k_{1}=k_{2}, i_{1}=i_{2}, \\ 0, & k_{1} \neq k_{2}, \\ 0, & i_{1} \neq i_{2} .\end{cases}$

In practice the number of measurement stations in rivers and estuaries is often large, so this assumption is not too unrealistic. The main object of this analysis is to apply Kalman filtering to a simplified problem that, however, still contains the essential feature of practical filtering problems. By solving the simplified problem, it becomes possible to expose the potential difficulties of more realistic Kalman filter applications.

Analogously to Eq. (5) we consider the Fourier integrals:

$$
\begin{aligned}
& \mathbf{F}_{i}^{k}=\int_{-0.5 \Delta x}^{0.5 \Delta x} \mathbf{F}^{k}(\ell) e^{j 2 \pi \ell i \Delta x} d \ell \\
& W m_{i}^{k}=\int_{-0.5 \Delta x}^{0.5 \Delta x} W m^{k}(\ell) e^{j 2 \pi \ell i \Delta x} d \ell \\
& W c_{i}^{k}=\int_{-0.5 \Delta x}^{0.5 \Delta x} W^{k}(\ell) e^{j 2 \pi \ell i \Delta x} d \ell \\
& Z_{i}^{k}=\int_{0.5 \Delta x} Z^{k}(\ell) e^{j 2 \pi \ell i \Delta x} d \ell \\
& V_{i}^{k}=\int_{-0.5 \Delta x} V^{k}(\ell) e^{j 2 \pi \ell i \Delta x} d \ell .
\end{aligned}
$$

In the above $\ell$ is the wave number. Substituting these integrals into Eq. (10) or (12) and then into Eq. (17) one has

$$
\begin{aligned}
& \mathbf{F}^{k+1}(\ell)=G(\ell) \mathbf{F}^{k}(\ell)+A(\ell)^{-1}\left[\begin{array}{l}
W m^{k}(\ell) \\
W c^{k}(\ell)
\end{array}\right] \\
& Z^{k}(\ell)=M \mathbf{F}^{k}(\ell)+V^{k}(\ell),
\end{aligned}
$$

where $G(\ell)$ is the amplification matrix of the finite difference scheme and, for the Preissmann scheme, $A(\ell)$ is defined by Eq. (6). For the Lax-Wendroff scheme we have $A(\ell)=I$. The covariance of the system and measurement noise are 
$Q(\ell)=\left[\begin{array}{cc}S m(\ell) & 0 \\ 0 & S c(\ell)\end{array}\right]$

$R(\ell)=S r$

Here $S m(\ell, k)=S m(\ell)$ and $S c(\ell, k)=S c(\ell)$ are defined by respectively the Eqs. (15) and (16). If the initial condition $P_{0}$ is given, the Kalman filter equations are employed to solve the filtering problem for each wave number separately (Maybeck 1979):

$P_{k}^{-}(\ell)=G(\ell) P_{k-1}^{+}(\ell) G(\ell)^{T}+A(\ell)^{-1} Q(\ell) A(\ell)^{-T}$

$P_{k}^{+}(\ell)=\left[I-\mathbf{K}_{k}(\ell) M\right] P_{k}^{-}(\ell)$,

where $P_{k}^{-}$and $P_{k}^{+}$are the covariances of the estimation error respectively before and after the $k$-th measurement. Furthermore:

$\mathbf{K}_{k}(\ell)=P_{k}^{-}(\ell) M^{T}\left[M P_{k}^{-}(\ell) M^{T}+S r\right]^{-1}$,

is the Kalman filter gain.

Given the model (23)-(26), questions of observability and controllability can be answered (Jazwinski 1970). It is a well-known fact that if a system model is both uniformly completely observable and controllable, the Kalman filter is uniformly exponentially stable (Maybeck 1979). Furthermore, we recall that if the original system is exponentially stable, the Kalman filter is also exponentially stable (Kwakernaak and Sivan 1972).

We now consider the system model (23)-(26) in more detail for both the LaxWendroff scheme and the Preissmann scheme in case the system noise is introduced each time step after the implicit finite difference equations have been solved (see Section 4). In both cases the matrix $A(\ell)^{-1}$ does not appear in Eq. (23). Employing the Preissmann scheme we have for waves with a length of $2 \Delta x$

$G\left(\frac{1}{2} \Delta x\right)=\left[\begin{array}{cc}-(1-\theta) / \theta & 0 \\ 0 & -(1-\theta) / \theta\end{array}\right]$,

and it can easily be verified that the water velocity is not observable. In this case the stability of the original system, introduced by choosing $0.5<\theta \leq 1.0$ have to guarantee the stability of the filter. Analogously, employing the Lax-Wendroff scheme we have for waves with a wave length of $2 \Delta x$ :

$G\left(\frac{1}{2} \Delta x\right)=\left[\begin{array}{cc}1-2 C r^{2}+\lambda \Delta t & 0 \\ 0 & 1-2 C r^{2}\end{array}\right]$,

and again the velocity component turns out to be not observable. However, in this case the sufficient condition for stability (9) guarantees the stability of the filter. Finally, for both the Preissmann and the Lax-Wendroff schemes, the amplification matrix for very long waves approaches:

$G(0)=\left[\begin{array}{cc}1-\lambda \Delta t & 0 \\ 0 & 1\end{array}\right]$.

Again, the velocity component is not observable. In this case bottom friction has to guarantee the stability of the filter.

To eliminate the effect of the initial condition we study the steady-state behaviour of the filter. We define: 

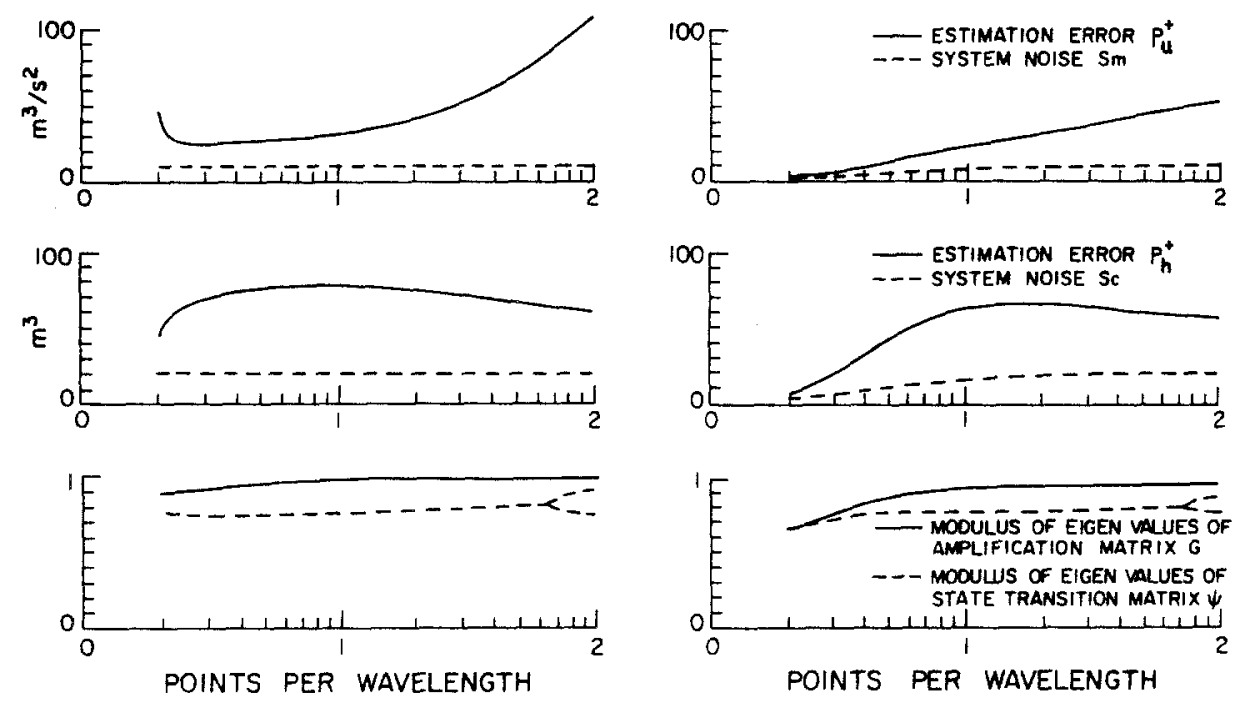

Figure 4

Figure 5

Figure 4. Steady-state filter performance using the Preissmann scheme with $\theta=0.53$ and with a bottom friction coefficient $\lambda=0.25 \times 10^{-6}$

Figure 5. Steady-state filter performance using the Preissmann scheme with $\theta=0.60$, a bottom friction coefficient $\lambda=1.0 \times 10^{-6}$ and spatially correlated system noise

$\mathbf{K}(\ell)=\lim _{k \rightarrow \infty} \mathbf{K}_{k}(\ell)$

and

$P^{+}(\ell)=\left[\begin{array}{ll}P_{u}^{+}(\ell) & P_{u h}^{+}(\ell) \\ \hdashline P_{u h}^{+}(\ell) & P_{h}^{+}(\ell)\end{array}\right]=\lim _{K \rightarrow \infty} P_{k}^{+}(\ell)$,

where $P_{u}^{+}(\ell)$ and $P_{h}^{+}(\ell)$ are the steady-state distributions of the estimation error of the water velocity and water-level just after a measurement has been incorporated. $P_{u h}^{+}(\ell)$ is the cross spectrum between these processes. $\overline{P_{u h}^{+}(\ell)}$ is the complex conjugate of $P_{u h}^{+}(\ell) . P^{+}(\ell)$ and $\mathbf{K}(\ell)$ can be determined by means of Eqs. (27)-(29). To gain insight into the stability of the filter we also compute the state transition matrix of the steady-state filter:

$\psi(\ell)=[I-\mathbf{K}(\ell) M] G(\ell)$,

and its eigenvalues $h_{1,2}(\ell)$.

In Fig. 4 the steady-state distributions $P_{u}^{+}(\ell)$ and $P_{h}^{+}(\ell)$ are shown as a function of the number of grid points per wave length $N=1 / \Delta x$ if the Preissmann scheme is used. The system noise has been chosen to be uncorrelated in space, yielding $S m(\ell)=S m$ and $S c(\ell)=S c$. These values are also shown in Fig. 4. 
The depth was chosen to be 10 meters while the effects of bottom friction $\left(\lambda=0.25 \times 10^{-6}\right)$ and numerical viscosity $(\theta=0.53)$ were taken into account in order to obtain an exponentially stable filter. Figure 4 shows that the uncertainty $P_{u}^{+}$for the number of grid points per wave length $N=2$ is rather large. As described above this is caused by the fact that this velocity component is not observable and $P_{u}^{+}$for $N=2$ is bounded only because of the numerical viscosity. The fact that $P_{h}^{+}$for $N=2$ is relatively small is caused by the same effect. Since the water velocity component does not affect the water-level, the appearance of the system noise $S m$ for $N=2$ does not increase the uncertainty $\boldsymbol{P}_{h}$ of this water-level component. Similar effects occur for the very long wave lengths. In this case bottom friction has to guarantee that $P_{u}$ for large $N$ is bounded. In Fig. 4 the modulus of the eigenvalues $g_{1,2}$ of the amplification matrix and $h_{1,2}$ of the state transition matrix are shown too. This figure illustrates that the filter is more stable than the original system. Unfortunately, this stability improvement turns out to be the least for very short waves.

In Fig. 5 we choose a spatially correlated system noise and increase the bottom friction coefficient $\left(\lambda=1.0 \times 10^{-6}\right)$ and the numerical viscosity $(\theta=0.60)$. As a consequence, $P_{u}^{+}$is decreased both for the long wave lengths as for the very short waves. Decreasing the uncertainty for the very short waves is essential since finite difference schemes are not able to represent short waves accurately. The existence of these short waves can cause unsatisfactory filter performance or may, in the non-linear case, introduce instabilities.

In Fig. 6 we consider the Preissmann scheme in which the system noise is introduced implicitly as described by Eq. (10) and not, as in the case of Figs. 4 and 5 each time step after the implicit finite difference equations have been solved. The system noise has been chosen to be uncorrelated in space. As can be seen from Fig. $6, P_{u}^{+}$and $P_{h}^{-}$behave in a similar way as in Fig. 5 and in this case, it is not necessary to assume that the system noise is spatially correlated. However, the filter is not controllable for very short waves. Despite the fact that $Q$ is positive definite, all the elements of $A^{-1}$, and consequently of the noise covariance $A^{-1} Q A^{-1}$, are zero for the short waves. Therefore, the eigenvalues of $P^{+}$for these waves become zero too. In practical filtering problems using a finite wordlength on the computer this may easily cause numerical problems.

Finally, Figure 7 demonstrates that similar results as in the case of Fig. 5 are obtained by using the Lax-Wendroff scheme instead of the Preissmann scheme.

In applying the filter to the system (23)-(26) the bottom friction coefficient as well as the noise statistics must be specified. However, these parameters are not known exactly. That is, the system model used in constructing the filter differs from the real system that generates the observations. It is clear that an inexact filter model degrades the performance of the filter. For the special filtering problem described in this section, we study this effect quantitatively. Suppose that the real system is described by:

$\mathbf{F}^{k+1}(\ell)=G_{r}(\ell) \mathbf{F}^{k}(\ell)+A_{r}(\ell)^{-1}\left[\begin{array}{l}W m^{k}(\ell) \\ W c^{k}(\ell)\end{array}\right]$,

$Z^{k}(\ell)=M \mathbf{F}^{k}(\ell)+V^{k}(\ell)$

where the covariances of the system and measurement noise are: 

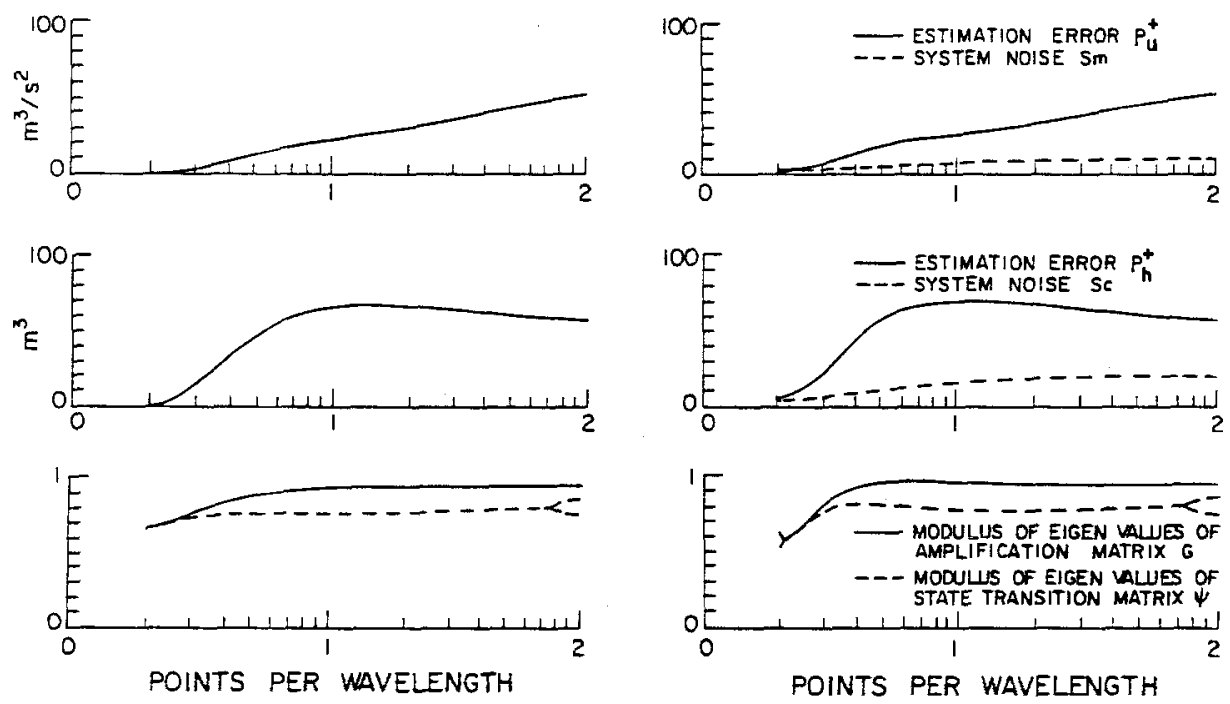

Figure 6

Figure 7

Figure 6. Steady-state filter performance using the Preissmann scheme with $\theta=0.60$, a bottom friction coefficient $\lambda=1.0 \times 10^{-6}$ and system noise introduced implicitly

Figure 7. Steady-state filter performance using the Lax-Wendroff scheme with bottom friction coefficient $\lambda=1.0 \times 10^{-6}$

$Q_{r}(\ell)=\left[\begin{array}{cc}S m_{r}(\ell) & 0 \\ 0 & S c_{r}(\ell)\end{array}\right]$

$R_{r}(\ell)=S r_{r}$

A measure of filter performance is provided by the estimation error covariance. The best performance of the filter is obtained when we model the real system by the correct Eqs. (36)-(39). Solving the filter equations results in the steady-state filter gain $\mathbf{K}_{r}(\ell)$ and estimation error:

$P_{r}^{+}(\ell)=\left[\begin{array}{ll}P_{u_{r}}^{+}(\ell) & P_{u h_{r}}^{+}(\ell) \\ \hline P_{u h_{r}}^{+}(\ell) & P_{h_{r}}^{+}(\ell)\end{array}\right]$

However, in practice, we model the system by the Eqs. (23)-(26) that differs from the real system described by the Eqs. (36)-(39). Solving the filter Eqs. (27)-(29) using this erroneous model yields the steady-state filter gain $\mathbf{K}(\ell)$ and estimation error $P^{+}(\ell)$.

The computed covariance matrix $P^{+}(\ell)$ is not the actual estimation error since the filter model differs from the real model. Neither produces this filter the optimal estimation error $P_{r}^{+}(\ell)$. The actual estimation error: 

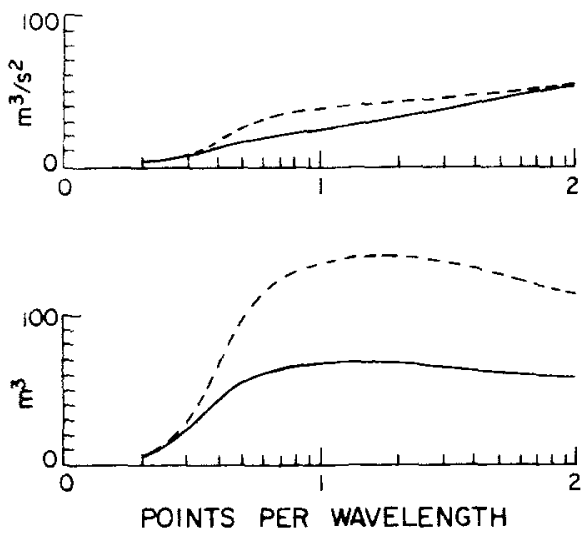

Figure 8a
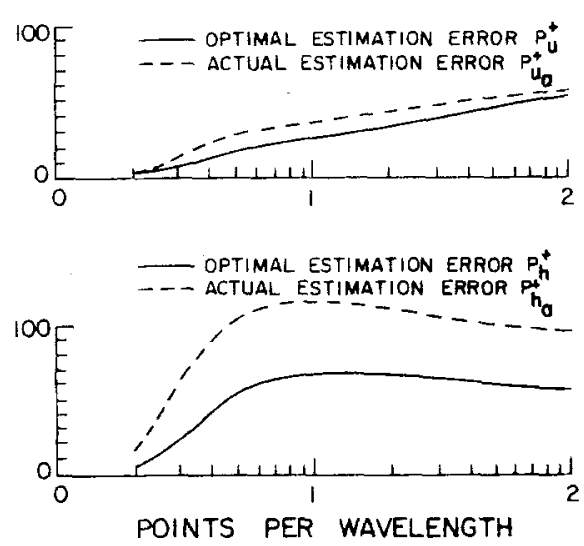

Figure 8b

Figure 8a. Steady-state filter performance in case the system noise is chosen too small

Figure 8b. Steady-state filter performance in case the system noise is chosen too large

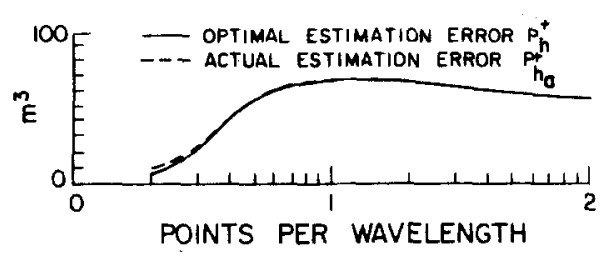

Figure 9. Steady-state filter performance using the first-order Lax-scheme instead of the secondorder Lax-Wendroff scheme

$P_{a}^{+}(\ell)=\left[\begin{array}{ll}P_{u_{a}}{ }^{+}(\ell) & P_{u h_{a}}{ }^{+}(\ell) \\ \overline{P_{u h_{a}}{ }^{+}(\ell)} & P_{h_{a}}{ }^{+}(\ell)\end{array}\right]$,

can be obtained by solving the equations:

$P_{a}^{-}(\ell)=G_{r}(\ell) P_{a}^{+}(\ell) G_{r}(\ell)^{T}+A_{r}(\ell) Q_{r}(\ell) A_{r}(\ell)^{T}$

$P_{a}^{+}(\ell)=[I-\mathbf{K}(\ell) M] P_{a}^{-}(\ell)[I-\mathbf{K}(\ell) M]^{T}+\mathbf{K}(\ell) S r_{r} \mathbf{K}(\ell)^{T}$.

In Fig. 8 the solutions of the Eqs. (42) and (43) are shown for some cases to study the effect of erroneous noise statistics. Here we employ the Lax-Wendroff scheme and assume that the real system behaviour is described by Fig. 7. The bottom friction coefficient $\lambda$ is again chosen to be $1.0 \times 10^{-6}$. The following situations are considered:

- The system noise is too small by an order of magnitude (see Fig. 8a): 
$S m(\ell)=0.1 S m_{r}(\ell) ; \quad S c(\ell)=0.1 S c_{r}(\ell) ; \quad S r=S r_{r}$

- The system noise is too large by an order of magnitude (see Fig. 8b):

$S m(\ell)=10 S m_{r}(\ell) ; \quad S c(\ell)=10 S c_{r}(\ell) ; \quad S r=S r_{r}$.

The main conclusions that are drawn from these figures are:

- The effect of choosing large incorrect system noise variance is small with respect to the large error that was introduced. This is of practical importance since the statistics of the system noise are often poorly known.

- Over-estimation of the system noise variance is less serious than underestimation.

Finally, we study the influence of the use of a finite difference scheme that possesses first-order accuracy. The scheme that is considered is the Lax scheme (Richtmyer and Morton 1967):

$\mathbf{F}_{i}^{k+1}=\left(\mathbf{F}_{i-1}^{k}+\mathbf{F}_{i+1}^{k}\right)-\frac{\Delta t}{2 \Delta x}\left(\mathbf{F}_{i+1}^{k}-\mathbf{F}_{i-1}^{k}\right)+\Delta t C_{2}\left(\mathbf{F}_{i-1}^{k}+\mathbf{F}_{i+1}^{k}\right)$,

with the amplification matrix:

$G(\ell)=\cos (2 \pi \ell \Delta x) I-j \frac{\Delta t}{\Delta x} C_{1} \sin (2 \pi \ell \Delta x)-\Delta t C_{2} \cos (2 \pi \ell \Delta x)$.

Again we assume that the real system is based on the Lax-Wendroff scheme with second-order accuracy and that it behaves as described in Fig. 7. Both the noise statistics as the bottom friction coefficient $\left(\lambda=1.0 \times 10^{-6}\right)$ are assumed to be known perfectly. However, we model the system using the Lax-scheme. The results shown in Fig. 9 indicate that the influence of the second-order terms of the finite difference equations is small compared to the effect of the choice of an incorrect system noise, except for very short waves.

\section{Conclusion}

In developing Kalman filtering procedures for numerical tidal models one is faced with questions of observability, stability and divergence of the filter, the numerical properties of the filter algorithm as well as the influence of the finite wordlength on the filter performance. In this paper we analyzed these aspects of Kalman filtering by deriving filters on the basis of the linear shallow water equations. Furthermore, we solved a specific filtering problem to expose the potential difficulties of the Kalman filter application. From the insights thus obtained we are able to compare different implementations with respect to the expected filter performance.

\section{Acknowledgements}

The author wishes to acknowledge the stimulating discussions with Dr. B. de Jong, P.G.J. ten Brummelhuis and Professor P.J. Zandbergen of the University of Twente.

\section{References}

Abbott, M.B. 1979: Computational Hydraulics. Pitman: London

Brummelhuis, P.G.J. ten.; De Jong, B.; Heemink, A.W. 1984: On-line prediction of water-levels in an estuary using Kalman filters. Proceedings of the 4th Int. Symp. on Stochastic Hydraulic. Urbana-Champaign, 153-166

Brummelhuis, P.G.J. Ten.; De Jong, B.; Heemink, A.W. 1988: A stochastic dynamic approach to predict water-levels in estuaries. J. of Hydraulic Engineering (to appear) 
Budgell, W.P.; Unny, T.E. 1980: A stochastic-deterministic model for predicting tides in branched estuaries. Proceedings of the 3rd Int. Symp. on Stochastic Hydraulics. Tokyo, 485-496

Budgell, W.P. 1986: Nonlinear data assimilation for shallow water equations in branched channels. J. of Geophysical Research. 91, 633-644

Goodson, R.E.; Klein, R.E. 1970: Definition and some results for distributed system observability. IEEE Trans. Autom. Control. 15, 165-174

Goodson, R.E.; Klein, R.E. 1971: Authors reply to: Comments on a definition and some results for distributed system observability. IEEE Trans. Autom. Control. 16, 106

Heemink, A.W. 1986: Storm surge prediction using Kalman filtering. Ph. D. Thesis, University Twente (also appeared as: Rijkswaterstaat Communications No. 46, Rijkswaterstaat, The Hague, 1986)

Heemink, A.W.; De Jong, B. 1982: The use of Kalman-Bucy filters in forecasting the water-levels in the Dutch coastal area. Proceedings of the 4th Int. Conf. on Finite Elements in Water Resour. Berlin: Springer-Verlag, 557-566

Jazwinski, A.H. 1970: Stochastic processes and filtering theory. New York: Academic Press

Kwakernaak, H; Sivan, R. 1972: Linear optimal control systems. New York: Wiley-Interscience

Liggett, J.A.; Cunge, J.A. 1975: Numerical methods of solution of the unsteady flow equations. In: Mahmoed, K.; Yevjevich, V. (eds.) Unsteady flow in open channels. Water Resour. Publications. Fort Collins, 89-182

Maybeck, P.S. 1979: Stochastic models, estimation and control. I. New York: Academic Press

Miller, R.N. 1986: Toward the application of the Kalman-Bucy filter to regional open ocean modelling. J. of Physical Oceanography. 16, 72-86

Parrish, D.F.; Cohn, S.E. 1985: A Kalman filter for two-dimensional shallow water model: formulation and preliminary experiments. National Meteorological Center, Office Note 304, DC 20233, Washington

Ritchmyer, R.W.; Morton, K.W. 1967: Difference methods for initial value problems. New York: Wiley-Interscience

Accepted April 10, 1988. 